\title{
SOBRE LA CONSTRUCCION ERGATIVA EN LA LENGUA GUATUSA
}

Adolfo Constenla U.

"Esta idea de que cualquier lengua conoce la relación sujeto-predicado, y con más precisión, presenta el mismo primer tiempo del análisis lingüístico de la experiencia, es un a priori que conviene rechazar desde un principio para que no afecte las condiciones de la observación".

André Martinet, La lingüistica sincrónica, p. 204.

0 . La presencia de sistemas de casos de tipo ergativo (los cuales se cuentan entre los temas que más discusión han originado en lingüística en la última década) en lenguas indígenas costarricenses como el guatuso, el bribri y el cabécar, había pasado totalmente desapercibida, incluso en investigaciones bastante recientes.

1. El primer estudio en que se detectó este tipo de construcción en una de las lenguas mencionadas fue mi análisis del guatuso (Constenla, 1975). En el mismo la presencia de la construcción ergativa se describe de la siguiente manera (pp. 264-265): "Para describir la integración de los constitutos objetivos es imprescindible aclarar que el guatuso es una lengua con un sistema de "casos" de tipo ergativo. Las lenguas con sistemas de casos de tipo ergativo se caracterizan frente a las que tienen sistemas de tipo nominativo (como el castellano) por asignar una misma forma a las expresiones que (empleando los términos de la gramática de las lenguas de tipo nominativo) funcionan como sujetos de las cláusulas intransitivas y objetos directos de las transitivas, en tanto que establecen una forma distinta para los sujetos de las cláusulas transitivas. Por ejemplo, en castellano se dice:
(a) Yosubo
(b) Tú subes
(c) Yo te subo
(ch) Tú me subes

Las formas correspondientes son en guatuso: (1)

(a) tó nataque
(b) pó mitaque (c) tonti pó mitaque

(ch) póti tó nataque

(la $n$ de ton 'yo' se pierde ante la inicial de nataque debido a una regla morfofonológica que no permite que dos consonantes idénticas entren en contacto).

Como puede notarse, lo que en las formas castellanas funciona como sujeto de la cláusula transitiva lleva en guatuso como característica el sujijo - $t i$ en tanto que lo que funciona en ellas como sujeto de cláusula intransitiva u objeto directo de cláusula transitiva no presenta ningún indicador de función, determinándose su relación con la forma verbal por medio de los prefijos indicadores de persona na- "primera persona" y $m i$ - "segunda persona".

De todo esto se concluye que en las lenguas como el guatuso la terminología sujeto-objeto directo no puede aplicarse, porque no refleja la manera en que se organizan las cláusulas. En su lugar se introducen los términos agente y objeto. Con el primero se designan las formas guatusas que se traducen por medio de sujetos de cláusulas transitivas castellanas; con el segundo, las que se traducen por medio de objetos directos de cláusulas transitivas o sujetos de intransitivas".

1.1. Los prefijos antes aludidos también se describieron en mi estudio de 1975 (pp. 276-277) de la siguiente manera:

"Prefijos de remisión a objeto (PO).

Esta subclase está integrada por cuatro miembros:

//na-// Remisión a los nombres de primera persona exclusiva: ton 'yo' y toi' 'nosotros'.

//ml-// Remisión al nombre de segunda persona pó 'tú' (y a su forma de plural pó maráma).

//I-// Remisión a los nombres de tercera persona.

//ma-// Este prefijo tiene un doble valor. Bási- 
camente indica remisión al nombre de primera persona inclusiva tótiqui 'nosotros', pero en ciertos constitutos sirve para indicar objeto indeterminado (impersonalidad).

La función de los prefijos citados es, en primer lugar, unirse a los elementos regentes de las formas pospositivas y de los constitutos objetivos para indicar la persona gramatical de los elementos regidos".

En las páginas 266 y 267 el término constitutivo objetivo se explicaba de la siguiente manera:

"Se denominan constitutos objetivos las frases resultantes de las construcciones exocéntricas objetivas de la lengua. Los elementos regentes de los constitutos objetivos son... formas verbales. Los elementos regidos son formas nominales que se denominan formas objeto...

La relación entre las formas regentes y las formas objeto se in dica por medio de los prefijos de remisión a objeto..."

El símbolo morfofonológico //I// se empleó para representar una regla de armonía vocálica que expliqué del siguiente modo:

\section{"Armonía vocálica}

El fenómeno de la armonía vocálica se da únicamente en tres morfemas monosilábicos... que funcionan como prefijos: //I-// 'remisión a objeto de tercera persona', //ml-// 'remisión a objeto de segunda persona', //r I-// 'reflexión o reciprocidad'. Estos morfemas presentan alternancias entre las vocales /a/ e /i/, que se representan por medio del símbolo //I// y obedecen a la siguiente regla:

//I// se realiza /a/ si está seguida por $/ / \bar{r} / /$ a su vez seguida por $/ / a / /$ o si la vocal precedente dentro de la misma palabra es $/ / a / /$. En los demás casos se realiza/i/".

Los siguientes ejemplos pueden ilustrar esta regla (cuánhe es una forma conjugada de cuáqui 'ver', rra- 'remisión a agente de primera persona exclusiva', rri- 'remisión a agente de tercera persona'):

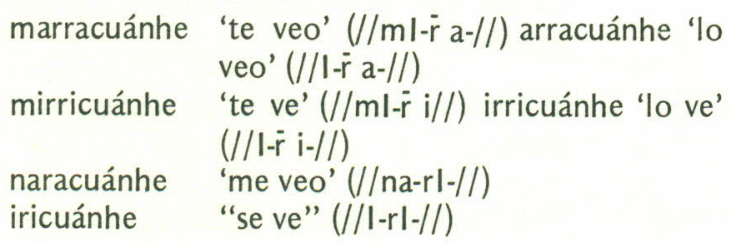

1.2. Al conjunto formado por la forma verbal transitiva, la frase objeto y la frase agente lo denominé expresión transitiva (p. 271):

"Las expresiones transitivas son formas resultantes de construcciones exocéntricas rectivas en las que los elementos regentes son constitutos objetivos y los elementos regidos expresiones de agente".

De acuerdo con sus componentes dividí las expresiones transitivas en dos tipos: aquellas en que (p. 271) la frase de "agente está integrada por una frase nominal y la relación entre ésta y el constituto objetivo que la rige se indica por medio del prefijo agentivo personal correspondiente a su persona gramatical..." y aquellas en que las frases de agente (p. 273) "están constituidas por frases resultantes de construcciones exocéntricas rectivas en las que el sufijo - $t i$ 'ergativo' funciona como elemento regente $y$ una forma nominal como elemento regido..." Una restricción característica de las últimas es que "la forma nominal regida por - $t i$ no puede ser el nombre de primera persona inclusiva tótiqui..." Por otra parte, en ellas "no intervienen los prefijos agentivos..."

1.3. Los prefijos de remisión a agente o agentivos constituyen una clase integrada por cuatro elementos que indican (p. 282) la persona de la forma nominal agente... "y que se presentan precedidos por los... de remisión a objeto". Los prefijos en cuestión según se describe en el lugar citado son:

rra- 'remisión a agente de primera persona exclusiva'

rrifa- 'remisión a agente de segunda persona' (con variantes facultativas rrif- y rripante las vocales y las consonantes sonoras no nasales, y las consonantes sordas, respectivamente).

rri'remisión a agente de tercera persona'

ri- 'remisión a agente de primera persona inclusiva'

De modo que a cada oración transitiva castellana corresponden dos posibilidades en guatuso, como se puede apreciar en los siguientes ejemplos: ton pó marracuánhe/tonti pó micuánhe 'yo te veo' pó tó narrifacuánhe/póti tó macuánhe 'tú me ves' 
En las páginas 284 y 285 de mi estudio aclaré que los prefijos de remisión a agente y a objeto pueden funcionar como sustitutos de las formas nominales a las cuales remiten, es decir, la presencia de éstas es facultativa, de modo que las oraciones que se acaban de citar pueden reducirse a:

marracuánhe/tonti micuánhe narrifacuánhe/póti nacuánhe

'yo te veo' 'tú me ves'

2. En los párrafos anteriores he tratado de resumir algunos aspectos del tratamiento que di a la ergatividad guatusa en 1975. En esta revista, se publicó posteriormente (Sánchez, 1979) un artículo sobre el mismo tema que en general coincide con mi estudio; no obstante, cabe señalar por lo menos las siguientes diferencias:

1. En ninguna parte del artículo de 1979 se señala que la presencia de $-t i$ y de los prefijos que remiten al agente es incompatible.

2. Tampoco hay mención en dicho artículo del hecho de que el elemento nominal de primera persona inclusiva no pueda entrar en secuencia con el sufijo-ti.

3. El concepto de sujeto es considerado por Sánchez como válido para la descripción del guatuso, en tanto que para él $(1979$, p. 71$)$ conviene hacer hincapié en la índole superficial del fenómeno de la ergatividad: 'determinada categoría de verbo exige cierta particularidad del sujeto, mientras que otra categoría verbal pide un comportamiento diferente del sujeto'.

En ningún momento da, sin embargo, razones en apoyo de su decisión de considerar lo que la lengua marca como trivial y de lo que la lengua no marca como lo realmente trascendente. Es en este último punto en el que su posición y la mía difieren radicalmente. En los siguientes párrafos haré explícitas algunas de mis razones para pensar que el concepto de sujeto no tiene lugar en ninguna descripción del guatuso que no se limite a forzar la lengua en moldes adoptados apriorísticamente sino que pretenda revelarnos la estructura propia de dicha lengua, y para pensar que la ergatividad no es ciertamente algo superficial en ella. El principio al cual me atengo en todo momento es que el establecimiento de una categoría en la des- cripción de una lengua sólo puede justificarse si dicha categoría corresponde apropiadamente a los hechos y los explica satisfactoriamente. El mero planteo de un historial derivativo de formas "latentes" a "patentes" ciertamente no constituye una explicación como algunos lingüistas parecen pensar.

\section{Las reglas de concordancia}

Toda gramática guatusa habrá de dar cuenta de la concordancia por medio de las dos series de afijos descritas. En una gramática construida con base en el concepto de sujeto, las reglas de concordancia deben ser innecesariamente complicadas ya que se tendrían que enunciar del siguiente modo:

a. El verbo de la oración transitiva concuerda con su sujeto por medio de los prefijos rra-, rrifa-, etc.

b. El verbo de la oración intransitiva concuerda con su sujeto por medio de los prefijos na-, $m i$-, etc.

c. El verbo de la oración transitiva concuerda con su objeto directo por medio de los prefijos na-, mi-, etc.

Nótese que se estaría caracterizando como dos concordancias diferentes lo que la lengua claramente nos indica por la identidad de los prefijos en b. y c. que se trata de una sola regla. Si nos preguntamos cuál es la base para hacer esto, es decir, para referirnos a algo como un sujeto en guatuso, no parece que pudiera darse otra respuesta que simple y llanamente la traducción española. (Sabemos que algo es sujeto porque traduce un sujeto del castellano). Pero, si ésta es la base, estaremos describiendo no la gramática guatusa sino sólo pretendiendo hacerlo cuando en realidad describimos la organización de las cláusulas castellanas.

Por lo contrario, una descripción basada en los conceptos de caso absolutivo y ergativo (lo que yo en 1975 Ilamé, respectivamente, objeto y agente), puede dar cuenta de los hechos de manera simple, directa y totalmente conforme con los hechos de la lengua. Las reglas de concordancia serían:

a. El verbo concuerda con el elemento nominal ergativo por medio de los prefijos de la serie rra-, rrifa-, etc. 
b. El verbo concuerda con el elemento nominal absolutivo por medio de los prefijos na-, mi-, etc.

\section{Cláusulas orientadas al ergativo}

En 1.2 se indicó que, como alternativa de las cláusulas transitivas en las que tanto la forma ergativa como absolutiva son frases nominales y el verbo concuerda con ambas, se dan otras (totalmente equivalentes desde el punto de vista referencial) en que la forma absolutiva es una frase nominal y el verbo sólo concuerda con ella, en tanto la forma ergativa es una construcción exocéntrica cuyos constituyentes son una frase nominal y el sufijo - $t i$. El primer tipo de cláusulas puede denominarse cláusulas transitivas no orientadas y el segundo tipo cláusulas transitivas orientadas al ergativo. Estas últimas constituyen la modalidad "marcada" y en consecuencia los entornos en que se pueden presentar son más restringidos (no se admiten por ejemplo en el modo imperativo). Su función en el discurso narrativo es la de llamar la atención sobre las formas ergativas que se refieren a participantes del hecho narrado que se presentan por primera vez o se reintroducen después de no figurar por un rato y que, al mismo tiempo son, tópicos de cláusulas que forman parte del hilo básico de la narración, esto es, de cláusulas de "primer plano" (Constenla, 1978). Como el sufijo -ti cumple, entre otras cosas, la función de marcar la presencia de un elemento nominal que aporta información nueva (o renovada) no es de extrañar que no se combine con el pronombre tótiqui (véase 1.2.) 'primera persona inclusiva' (es decir 'vosotros o tú y yo'). No parece posible que un tópico integrado por el hablante y el oyente $u$ oyentes pueda constituir información nueva en un contexto narrativo referido al pasado. Esta restricción no es igualmente válida en otros tiempos, sin embargo el guatuso la ha fijado para todos los casos.

Volviendo al tema que nos ocupa, en una gramática basada en los conceptos de absolutivo y ergativo todo lo anterior es muy fácil de describir. El elemento -ti sólo se da en las formas ergativas. En una gramática basada en el concepto de sujeto no sucede lo mismo. La aparición de -ti se tendrá que definir no por referencia a este concepto solamente, sino que habrá que decir que este elemento entra sólo en la formación de sujetos de verbos transitivos. Es decir, nuevamente, nos enfrentaremos al problema de que la lengua trata como cosas distintas e inidentificables el "sujeto" del verbo trassitivo y el "sujeto" del verbo intransitivo, es decir el ergativo y el absolutivo.

\section{Otras construcciones}

El examen general de las construcciones sintácticas guatusas nos comprueba la misma situación: para describirlas el concepto de sujeto resulta totalmente innecesario, como en los dos casos siguientes:

5.1. En guatuso existe una clase de verbos derivados de otros por medio de un sufijo -tequi. Dichos verbos significan acontecimientos en que el agente es desconocido, como en el caso de corrtequi 'golpearse (accidentalmente)', cuactequi 'verse', etc. La derivación se efectúa sólo a partir de verbos transitivos (es decir no se puede unir -tequi a verbos intransitivos para producir equivalentes de las formas castellanas como "se baila', 'se llora'). Si consideráramos las oraciones guatusas como tó nacorrtenhe 'yo me golpeé (accidentalmente)' como derivada en algún sentido de oraciones del tipo de naíti tó nacorróye 'aquel me golpeó', la noción de sujeto a secas nos resultaría de escasa ayuda, pues la derivación se produce únicamente por medio de la supresión no de cualquier "sujeto", sino de un "sujeto" de verbo transitivo, es decir, de una forma ergativa.

5.2. En guatuso se da el tipo de construcción denominado antipasiva. Esta construcción se forma únicamente a partir de los verbos transitivos a los cuales se une el prefijo fa- $\sim f-\sim p$ - 'antipasivador'. El participante que en las oraciones transitivas está representado por la forma absolutiva, en estas oraciones es una frase posposicional facultativa construida según el verbo de que se trate, a partir de una de las siguientes posposiciones: $y u$ 'con', Iha 'sobre', co 'en'. El participante que en las oraciones transitivas está representado por la forma ergativa es en la antipasiva una forma absolutiva. Compárense las siguientes oraciones por ejemplo:

\begin{tabular}{|c|c|c|}
\hline $\begin{array}{l}\text { ochápacati } \\
\text { hombre-erg. }\end{array}$ & $\begin{array}{l}\text { pó } \\
\text { te }\end{array}$ & $\begin{array}{l}\text { mierre } \\
\text { 2a. disparó }\end{array}$ \\
\hline $\begin{array}{l}\text { ochápaca } \\
\text { hombre }\end{array}$ & $\begin{array}{l}\text { (po milha) } \\
\text { ti } 2 \text { a. hacia }\end{array}$ & $\begin{array}{l}\text { iferre } \\
\text { disparó }\end{array}$ \\
\hline
\end{tabular}

(las posposiciones concuerdan con sus términos, de ahí la presencia de $m i$ - en milha). 
Nuevamente tenemos un tipo de derivación que afecta, no a cualquier "sujeto", sino únicamente al "sujeto de oración transitiva", o sea al caso ergativo.

\section{Conclusión}

De acuerdo con la argumentación precedente, queda claro que en guatuso, en tanto la noción de sujeto no parece tener pertinencia alguna, las nociones de ergativo $y$ absolutivo son absolutamente indispensables para describir y explicar la morfosintaxis. ¿A qué se podría atribuir entonces el que un lingüista proclame que lo que no se muestra por ninguna parte en la lengua es lo realmente importante y que lo que se muestra por todas partes no lo es? Esto sin lugar a dudas se debe en mucho al papel tan importante que desafortunadamente durante los últimos treinta años han tenido en la lingüística algunas teoríac sue determinan una aproximación apriorística y prejuicidada al objeto de estudio. Las consecuencias de esta actitud son bien graves. Como lo señaló Hockett (1966, p. 70) refiriéndose al trabajo de una de estas corrientes, la tagmemática, pero con palabras que con pequeños cambios podrían aplicarse también, por ejemplo, a la gramática transformacional:

"No usan su técnica matriz para descubrir las 'categorías' que realmente funcionan en la lengua: por lo contrario, se aproximan con un conjunto ya hecho de 'categorías' que la lengua con seguridad debe tener y por medio de la permutación de matrices intentan descubrir como estas 'categorías' están representadas por partes de palabras. Me opongo violentamente. No es así como se descubren los mecanismos de una lengua particular, y, por fuerza, esta aproximación a las lenguas individuales nos impide definitivamente el descubrir inductivamente universales lingüísticos".

\section{NOTAS}

(1) He reemplazado la transcripción fonemática por una práctica. El valor fonético de los símbolos empleados es el siguiente:

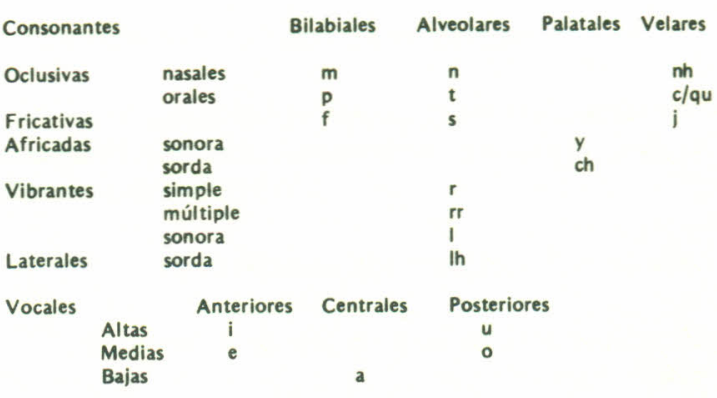

Las vocales largas se consideran como resultado de la suma de un fonema de cantidad extra a las vocales. Dicho fonema se representa con la tilde (').

\section{BIBLIOGRAFIA}

Constenla Umaña, Adolfo. 1975. "La lengua guatusa: fonología, gramática y léxico". Tesis de licenciatura, Universidad de Costa Rica.

. 1978. "Voice and the foregrounding of new topics in Guatuso". Trabajo sin publicar. Universidad de Pensilvania.

Hockett, Charles F. 1966. "What algonquian is really like". International Journal of American Linguistics, 32: 59-73.

Sánchez Corrales, Víctor Manuel. 1979. "El maleku: lengua ergativa" Revista de Filología y Lingüistica de la Universidad de Costa Rica, 5(1-2): 67-71. 
